\title{
IL Dialogo della Rettorica di Sperone Speroni e la sua Traduzione in Portoghese
}

\author{
Ana LuZa Leite Bado* \\ Sergio Romanelui*
}

ABSTRACT: In questo articolo presentiamo al pubblico brasiliano, interessato alla storia della lingua italiana, un testo inedito in lingua portoghese scritto da uno dei personaggi che presero parte alla "questione della lingua" fra Quattrocento e Cinquecento in Italia. Si tratta del Dialogo della Rettorica di Sperone Speroni. Ci sembra rilevante sottolineare che, seppur molto citata e studiata, la "questione" sempre venga affrontata facendo riferimento solo ad alcuni dei suoi protagonisti, ovvero Pietro Bembo, Giangiorgio Trissino, Baldassar Castiglione e Machiavelli, dimenticando però che coinvolse tutti i principali intellettuali dell'epoca (e non solo se pensiamo che fino a Gramsci molti intellettuali hanno scritto sulla "questione") e che diede origine a una serie di interessanti testi di pregevole qualità linguistica, storica e letteraria. Per questo motivo e nella speranza di risolvere questa lacuna, presentiamo qui il testo tradotto in portoghese inserendolo nel più ampio dibattito sulla lingua.

PAROLE CHIAVE: Sperone Speroni; lingua italiana; questione della lingua.

RESUMO: Neste artigo apresentamos ao público brasileiro, interessado na história da língua italiana, um texto inédito em língua portuguesa escrito por um dos personagens que participaram da "questione della língua" entre o século XV e o século XVI na Itália. Trata-se do Dialogo della Rettorica de Sperone Speroni.

* Graduanda do curso de Língua e Literatura Italiana na Universidade Federal de Santa Catarina e bolsista do CNPq.

** Docente do Departamento de Língua e Literatura Estrangeiras e da PGET (Programa de Pós-Graduação em Tradução) da Universidade Federal de Santa Catarina e Bolsista em Produtividade do CNPq. 
Parece-nos relevante destacar que, ainda que muito estudada e citada, a "questione" sempre é abordada fazendo referência somente a alguns de seus protagonistas, ou seja, Pietro Bembo, Giangiorgio Trissino, Baldassar Castiglione e Machiavelli, esquecendo-se, porém, que envolveu todos os principais intelectuais da época (e não somente, se pensarmos que até Gramsci muitos intelectuais italianos escreveram sobre a "questione") e que redundou numa série de textos interessantes e de grande qualidade linguística, histórica e literária. Por essa razão, no intuito de preencher essa lacuna, apresentamos aqui o texto traduzido para o português inserindo-o no mais amplo debate acerca da língua.

PALAVRAS-CHAVE: Sperone Speroni; língua italiana; questione della lingua.

ABSTRACT: In this paper we present to the Brazilian public, interested in the history of the Italian language, a text unpublished in Portuguese written by one of the intellectuals who took part in the "questione della lingua" between the fifteenth and sixteenth centuries in Italy. This is the Dialogo della Retorica by Sperone Speroni. It seems important to emphasize that, although much quoted and studied, the "questione" still is addressed by referring only to some of its protagonists, namely Pietro Bembo, Gian Giorgio Trissino, Baldassare Castiglione and Machiavelli, forgetting that it involved all the leading intellectuals of the time (and not just them, when we think that many intellectuals until Gramsci wrote about the "questione"), and that resulted in a number of interesting texts of excellent linguistic, historical and literary quality. For this reason, and in the hope of resolving this gap, we present here the text translated into Portuguese by inserting it into the wider debate about the language.

KEYWORDS: Sperone Speroni; Italian language; questione della lingua. 
122 ottobre del 2013 abbiamo avuto il piacere di presentare al pubblico brasiliano il secondo volume dei Clássicos da Língua Italiana (il primo è uscito nel 2012), contenente dei testi inediti di Sperone Speroni, tradotti per la prima volta in portoghese brasiliano. L'autore scelto e tradotto in questa seconda edizione espresse le sue riflessioni sulla querelle fra la lingua italiana e i volgari, nella cosiddetta "questione della lingua", attraverso una serie di dialoghi, in particolare nel Dialogo delle lingue e nel Dialogo della Rettorica. In questo secondo volume presentiamo quindi la traduzione di quest'ultimo, assieme alla traduzione di alcune parti tratte dell'interessante biografia, ancora inedita in Brasile, di Sperone Speroni, scritta da Francesco Cammarosano, sicuri che entrambi i testi possano diventare utili strumenti di ricerca per tutti coloro che sono interessati alla storia della lingua italiana.

Come si è detto, questo è il secondo di una serie di volumi frutto del lavoro dei membri che 1 Testo tradotto in portoghese e pubblicato nel volume Clássicos da Tradução della PGET-UFSC. 
fanno parte del gruppo di ricerca registrato presso il CNPq, Estudos Linguísticos e AquisiçãoAprendizagem do italiano como $L E$, e in particolare del progetto di ricerca intitolato Projeto de tradução dos clássicos da polêmica quinhentista acerca da língua italiana ou Questione della Lingua, in corso dal 2009 presso il Departamento de Língua e Literatura Estrangeiras dell'Universidade Federal de Santa Catarina e il cui obiettivo è raccogliere e tradurre, per la prima volta in lingua portoghese del Brasile, alcuni testi fondamentali sulla "questione della lingua italiana" ${ }^{2}$.

La traduzione di Sperone Speroni, oggetto del secondo volume e di quest'articolo, è risultato del lavoro di ricerca della laureanda Ana Luiza Leite Bado e di Sergio Romanelli, con borsa PIBIC/CNPq, concluso nel 2012 e intitolato Tradução dos clássicos da língua italiana: Sperone Speroni.

Sperone Speroni (Padova, 1500-1588) fu un umanista, letterato e filosofo che dedicò grande parte della sua vita a scrivere dialoghi che venivano discussi nei circoli letterari dell'epoca. Tra questi dialoghi, che risalgono al periodo che va dal 1542 al 1558, troviamo, come detto, il Dialogo delle Lingue ed il Dialogo della Rettorica. Entrambi, anche se in modo diverso, trattano della "questione della lingua", avvenuta nel corso del Rinascimento italiano.

Il dialogo era un genere testuale e letterario molto in voga in Italia fra il Quattrocento e il Cinquecento. Veniva usato per argomentare su una determinata questione il più delle volte polemica. Attraverso il dialogo l'autore forniva una risposta immediata alle tesi degli avversari, senza esporsi in prima persona (spesso difatti si "nascondeva" dietro a uno dei vari personaggi che partecipavano alle discussioni). Era un gioco di contrappunti che raggiungeva lentamente il suo scopo, ossia, smantellare la tesi contraria con una serie di ragionamenti, sillogismi anche piuttosto raffinati, permettendo però al lettore di avere un quadro ampio sull'argomento e sulle parti in causa. I dialoghi potevano essere di due tipi: un primo tipo di stampo aristotelico era più legato alla difesa e alla dimostrazione di una singola tesi (frequentemente quella dell'autore del libello); un secondo, di stampo platonico, in cui l'autore rinunciava alla sua posizione personale a favore della discussione e dell'argomentazione di tutte le tesi coinvolte. Rientrano appunto in questa seconda tipologia il Dialogo delle Lingue e il Dialogo della Rettorica. In entrambi i dialoghi gli argomenti dei personaggi si alternano senza l'apparente e diretta interferenza dello scrittore, offrendo al lettore, presentato come una sorta di spettatore extradiegetico, la possibilità e il privilegio di presenziare alla costruzione del discorso per poter costituire la propria opinione e seguire le argomentazioni proposte dall'esterno.

2 Il gruppo, coordinato da Sergio Romanelli, prevede la partecipazione di vari docenti appartenenti alle seguenti università: Universidade Federal de Santa Catarina (Sergio Romanelli e Carolina Pizzolo Torquato); Universidade de São Paulo (Cecilia Casini e Elisabetta Santoro); Universidade Federal de Minas Gerais (Patrizia Collina Bastianetto e Lucia Fulgencio); Università Ca' Foscari di Venezia (Paolo Torresan) e Università Gabriele D’Annunzio di Pescara (Cecilia Santanché). Partecipano, inoltre, laureandi, studenti del master di ricerca e di dottorato del corso di Língua e Literatura italiana e delle scuole di master e dottorato delle tre università brasiliane legate al progetto. 
Sperone Speroni svolse un importante ruolo nel corso del dibattito sulla "questione della lingua" e fu uno dei più noti difensori del volgare.

Riprendendo molto brevemente il contesto in cui si inseriscono le opere di Speroni, si deve ricordare che è possibile far risalire la "questione della lingua" al XIV secolo quando, nel De Vulgari Eloquentia, Dante Alighieri si era schierato a favore delle lingue volgari (sebbene quest'opera sia stata pubblicata solo due secoli più tardi). In questo trattato Dante, che anticipava con una certa preoccupazione il problema della varietà linguistica esistente nel territorio italiano, aveva sviluppato una tesi in difesa del volgare illustre il quale, secondo il poeta fiorentino, avrebbe dovuto riunire ciò che esisteva di migliore fra i principali dialetti italiani, dando vita ad una specie di spirito comune e universale comprendente le differenti varianti presenti nella penisola italiana. Con la diffusione del fiorentino erudito delle Tre Corone (Dante, Petrarca e Boccaccio), ampiamente usato dagli autori del XV secolo, la necessità di un'unità linguistica si fece ancora più forte e il dibattito sulla "questione della lingua" vide la partecipazione di molti intellettuali dell'epoca. In quel periodo la situazione linguistica della Toscana era frammentaria. Da un lato, esistevano i difensori di una lingua arcaica, il cosiddetto fiorentino erudito, che sarebbe servito per "restaurare l'unità linguistica risalente al latino lingua madre" (ROMANELLI, 2012, p. 7). Dall'altra parte, vi erano i difensori di un'ipotetica lingua cortigiana o italica, ovvero, di una lingua più adeguata alla vita civile dell'uomo di corte, una lingua aperta all'inserimento di parole straniere, sia spagnole che francesi (TESI, 2007). Ad esempio, la lingua cortigiana sostenuta dal Castiglione si fondava su di un modello linguistico strettamente connesso alla sua funzione sociale, per questo motivo censurava l'uso di parole e di stili sintattici ritenuti inappropriati alla naturalità della conversazione colta (Ibid.). Diversamente da Castiglione, Machiavelli difendeva il fiorentino popolare e parlato, mentre Pietro Bembo era il massimo rappresentante della difesa del fiorentino letterario.

Tra i partecipanti a questo dibattito ci sembra opportuno ricordare le posizioni assunte da Baldassar Castiglione, Giangiorgio Trissino e appunto Sperone Speroni.

Baldassar Castiglione (1478-1529), che rivendicava il diritto di ciascuno a parlare nella propria lingua, sosteneva la creazione di una lingua che non eliminasse i latinismi, i neologismi e neanche i vari dialetti parlati in Italia. Giangiorgio Trissino (1478-1550), basandosi sul De Vulgari Eloquentia, trattato che a sua volta aveva tradotto, fu il primo a occuparsi della "logica intima dell'annosa questione in ciò che essa ha di più consistente e valido sul piano storico" (SOZZI, 1976, p. 84), ossia, del principio di italianità della lingua. Sperone Speroni si opponeva 
invece al formalismo di Pietro Bembo quando affermava che l'imitatio - assai praticata durante la prima fase dell'Umanesimo, periodo in cui la lingua latina ancora dominava (FURLAN, 2006) - non fosse da ritenere una pratica artistica tanto meno un atto di intelligenza. Lo scrittore del Dialogo della Rettorica affermava che le parole sono come uno specchio, ogni parola riflette il suo tempo e per questo gli scritti del 1300 non potevano riflettere la modernità del pensiero cinquecentesco.

Il Dialogo della Rettorica, oltre a rivendicare il valore sociale della retorica, è un trattato che parla anche dei valori e della condotta morale degli esseri umani e introduce la figura del nuovo letterato, capace di argomentare in lingua volgare. Il Dialogo si svolge fra tre personaggi: Valerio, Brocardo e Soranzo. Soranzo è per l'appunto il primo a intervenire sulla "questione":

Oltra di questo io sono in dubbio, se l'arte oratoria della lingua Latina si convegna con l'altre lingue, specialmente con la Toscana che noi usiamo oggidì: nella quale io ho opinione che a dilettare alcun malinconico, imitando il Boccaccio, qualche novella si possa scrivere senza più cosa veramente diversa dalle tre guise di cause, le quali da' Latini scrittori sola e generale materia della loro arte rettorica si nominarono." (SPERONI, 1978, pp. 203-204)

Além disso, eu estou em dúvida se a arte oratória da língua latina é condizente com as outras línguas, especialmente com a toscana que nós usamos atualmente; eu sou da opinião que algum saudoso de Boccaccio possa se deleitar escrevendo nela alguma novela sem problemas; sendo esse fato na verdade diferente dos três tipos de causas que foram nomeadas pelos escritores latinos como matéria única e geral de sua arte retórica. ${ }^{3}$

In questo brano è possibile osservare un riferimento diretto a Pietro Bembo quando Soranzo dice: "alcun malinconico, imitando il Boccaccio". Lo scrittore allude alla proposta nostalgica di Bembo che consisteva nell'imitare la lingua italiana usata da Petrarca e Boccaccio, lingua che, secondo Bembo, aveva raggiunto una perfezione insuperabile. Questa presunta perfezione, ma anche il fatto che il fiorentino fosse capace di rappresentare una borghesia in crescita, e che "non si vedeva rappresentata né dall'opzione aristocratica dal latino, né dall'opzione popolare del fiorentino parlato" (ROMANELLI, 2012, p. 9), fece sì che l’idea del Bembo definisse alla fine la polemica questione. Tommaso Sozzi (1976) aggiunge che la soluzione di Bembo era

3 Per mostrare il lavoro di traduzione da noi fatto, lasceremo sempr le traduzioni in portoghese sotto il testo originale in italiano. 
però inefficiente soprattutto sul piano linguistico, perché ignorava un fattore primario in ogni lingua: l'oralità. Speroni aveva già citato la posizione del Bembo nel Dialogo delle Lingue: "lodo sommamente la nostra lingua volgare, cioè toscana $[. .$.$] toscana dico, non la moderna$ che usa il vulgo oggidì, ma l'antica, onde sì dolcemente parlino il Petrarca e il Boccaccio" (SPERONI, 2006, p. 221); dove affermava che le lingue volgari, come il lombardo, non sono adatte al discorso e alla poesia. In questo modo manifestava il proprio disaccordo quanto alle affermazioni del Cortegiano nel libro di Castiglione, il quale credeva alla necessità di portare il pensiero scientifico e filosofico alla plebe dicendo a riguardo: "sarà proprio degli amatori e studiosi delle dottrine, le quali hanno ricetto non nelle lingue ma negli animi d'i mortali" (SPERONI, 2006, p. 248).

Ritornando al discorso di Soranzo, va detto che lui non crede nella capacità argomentativa della lingua volgare - e questo lo pone sullo stesso piano di Pietro Bembo - ed è contrario all'ideale di Baldassar Castiglione, il quale affermava che la lingua italiana doveva formarsi senza escludere i latinismi e gli altri dialetti parlati nella penisola. D'altronde, Castiglione chiedeva che ognuno potesse parlare e scrivere nella propria lingua (SOZZI, 1976). Ne Il Cortegiano, pubblicato nel 1528, Baldassar Castiglione dedica alla "questione della lingua" i capitoli cha vanno dal XXVIII al XXXIX. In particolare nel trentottesimo capitolo difende la lingua come espressione del pensiero individuale e nel capitolo seguente sostiene la sostanza del pensiero contro il formalismo linguistico. Il pensiero di Castiglione, nel Dialogo delle Lingue, appare un'altra volta nel discorso del Cortegiano, si veda a questo proposito il brano che segue:

Almeno dirò quello che io averò in core; e lo studio che io porrei in infilzar parolette di questo e di quello, sì lo porrò in trovare e disporre i concetti dell' animo mio, onde si deriva la vita della scrittura; ché male giudico potersi usare da noi altri a significare i nostri concetti quella lingua, tosca o latina che ella si sia, la quale impariamo e esercitiamo non ragionando tra noi i nostri accidenti, ma leggendo gli altrui. [...] Non dico però che uomo scriva né padovano né bergamasco, ma voglio bene che di tutte le lingue d'Italia possiamo accogliere parole e alcun modo di dire. (FURLAN, 2006, p. 224)

L'idea di Castiglione appare frequentemente nei dialoghi di Sperone Speroni perché entrambi sono contrari al canone dell'imitazione e al formalismo di Bembo, come chiarisce Sozzi in 
questo passo:

Nell'opzione per la culturalità sostanziata di pensiero di contro al formalismo linguistico e nella rivendicazione [...] dei diritti dell'originalità contro il canone pedantesco dell'imitazione lo Speroni emerge, accanto al Castiglione [...] come oppositore del formalismo bembistico. (1976, p. 87)

In modo specifico, nel Dialogo della Rettorica, il discorso di Brocardo è significativo in quanto, attraverso questo, l'autore svela la sua posizione. Il personaggio che frequentemente si sofferma a disquisire sulla natura delle parole, difende a più riprese la capacità argomentativa della lingua volgare: "e da' scogli delle parole Latine, nelle quali a lungo andare il parlamento si romperebbe, bellamente mi guardarò; a più faggio nocchiero di me lasciando la cura di dover fare sì periglioso viaggio." ["Com muito cuidado, evitarei usar as palavras latinas que a longo prazo prejudicariam a construção da minha fala, deixando essa viagem no perigoso mar a um navegador mais hábil que eu."] (SPERONI, 1978, p. 208). Brocardo evita l'uso di parole latine perché la lingua volgare, secondo lui, è in grado di valorizzare il suo discorso ed anche perché è la sua lingua, quella che meglio domina. Analogamente Baldassar Castiglione ne Il Cortegiano afferma chiaramente che preferisce essere riconosciuto come un lombardo, quando parla lombardo, piuttosto che essere riconosciuto come non toscano, per il fatto di parlare un toscano "eccessivo". Meglio una parlata naturale che esprime chiaramente i pensieri, che una parlata artificiale, molto più preoccupata delle regole che causa stanchezza in chi parla e in chi ascolta (BASTIANETTO, 2012).

Il Brocardo cita come esempio il successo di Petrarca che, a parer suo, era stato raggiunto solo perché lui era toscano e conosceva bene la propria lingua:

Ma perchè il tutto sappiate; soleva dirmi Messer Trifone, che al Petrarca l'esser nato Toscano e saper ben la sua lingua, ed in contrario il non saper la Latina, benchè l'arte tenesse, fu cagione di farlo grande nell'una, ma nell'altra molto manco che mediocre. (SPERONI, 1978, p. 220).

Mas porque saibam tudo, costumava me dizer o Senhor Trifone que, o fato de Petrarca ter nascido toscano e conhecer bem a sua língua, e, ao contrário, não 
saber a latina, apesar de usar seu estilo, foi motivo para torná-lo grande em uma, mas na outra muito menos que mediocre.

Significativo a questo proposito è il discorso del Magnifico nel libro di Castiglione. Il personaggio afferma che se Petrarca e Boccaccio fossero ancora vivi neanche loro ricorrerebbero al toscano antico, dato che si tratta di una lingua che si serve di parole inadeguate ai costumi della sua epoca. Sorge allora un nuovo filo che collega il pensiero di Speroni a quello di Baldassar Castiglione: entrambi possono essere considerati moderni in quanto capiscono che la lingua, come un organismo vivo, è soggetta a cambiamenti temporali. Ci pare altresì che entrambi gli autori riconoscano il valore dei dialetti, i quali, pur avendo una minor frequenza d'uso, sono delle lingue a tutti gli effetti e sono rette dai propri sistemi lessicali e morfosintattici.

Tornando alla "questione della lingua", si può affermare che Sperone Speroni, secondo gli ideali esplicitati nel Dialogo delle Lingue e nel Dialogo della Rettorica, era a favore del fiorentino erudito della sua epoca. Principalmente nel Dialogo delle Lingue l'autore, ribadendo ancora una volta la sua posizione contro i nostalgici latinisti, dichiara apertamente che "l'Italia era capace d'esprimere con dignità e bellezza non solo tutto ciò che v'era nella poesia ma ancora nella storia, nelle scienze e nell' arti”' (SPERONI, 1978, p. 13). Per lo scrittore dei dialoghi era essenziale difendere vigorosamente l'idea che il volgare poteva e doveva essere usato in qualsiasi ambito scientifico e culturale, anche nell'educazione.

Dalle idee proposte da Speroni si evince che l'autore sperava non solo nella valorizzazione del fiorentino erudito usato nel Rinascimento, ma anche che l'uso del volgare fosse significativo nell'espressione dei concetti delle nostre anime, in quanto questi devono essere rivelati usando le nostre parole e non mediante ideali che appartengo ad altri. In questo modo l'autore rendeva chiara la sua posizione anti-umanista, rifiutando la soluzione di Pietro Bembo e concordando in molti aspetti col pensiero di Baldassar Castiglione.

Per questo motivo il testo di Speroni non può essere interpretato senza tener conto del contesto nel quale l'autore si trova inserito. L'annosa questione (non solo linguistica, ma anche culturale, storica e politica) in cui lo scrittore del Dialogo della Rettorica intervenne, fu risolta solo nel 1861, con l'unità politica d'Italia.

Il principe dell'Accademia degli Infiammati, escluso in maniera, a nostro avviso, ingiusta dagli studi della storia della lingua italiana, ha avuto il merito di essersi interrogato, in volgare, sulla questione del potere argomentativo delle lingue volgari e di essersi chiesto se potevano queste 
avere lo stesso peso della lingua latina. Infine, ed è proprio questa la domanda trasversale che fa da sfondo a tutto il dibattito sulla "questione della lingua", l'autore si domanda quali fra le lingue parlate nella penisola sarebbe stata la più qualificata ad assumere lo status di una lingua nazionale.

Prima di concludere, riportiamo alcuni chiarimenti sui criteri che hanno guidato la nostra traduzione del testo di Speroni. La distanza storica, linguistica e culturale del testo ci ha portati a riformulare la morfosintassi e la punteggiatura per renderlo più leggibile e comprensibile al lettore brasiliano. Tuttavia, abbiamo anche cercato di mantenere, quando possibile, la peculiare costruzione dell'autore, evidentemente influenzata dal latino. In altre parole, abbiamo scelto una strategia di traduzione ibrida, a metà fra la domesticazione e la stranierizzazione. Sebbene l'obiettivo sia, infatti, avvicinare il testo al lettore contemporaneo, crediamo sia importante anche mantenere la percezione delle peculiarità formali del testo originale, soprattutto in testi di questo tipo che devono rivelare al lettore straniero una lingua, quella italiana, in statu nascendi, ancora indefinita e appunto ibrida. Per quanto riguarda i nomi dei personaggi e dei luoghi dell'Antichità e dell'Italia Rinascimentale citati da Speroni, quando sono stati trovati equivalenti in portoghese, sono stati tradotti, quando questo non è stato possibile, si è mantenuta la forma italiana con nota in calce esplicativa nei casi in cui ciò era necessario.

A fini didattici abbiamo messo note in calce per fornire informazioni su autori, personaggi e termini non comuni. Speriamo in questo modo di essere riusciti nell'intento di mettere a disposizione di ricercatori, studenti e lettori brasiliani un testo fondamentale ai fini della comprensione di una questione così importante per laformazione della lingua e della cultura italiana.

\section{Riferimenti bibliografici}

BASTIANETTO, P. Introdução. In: ROMANELLI, S. Antologia Bilíngue. Clássicos da Língua Italiana. Tubarão: Copiart; Florianópolis: PGET/UFSC, 2012, p. 96-101.

FURLAN, M. (org.). Antologia Bilíngue. Clássicos da Teoria da Tradução. Vol. 4 Renascimento. Florianópolis: UFSC/NUPLITT, 2006.

MACHIAVELLI, N. Discorso, O Dialogo Intorno alla Nostra Lingua. Edizione critica con introduzione, note e appendice a cura di Bortolo Tomasso Sozzi. Torino: Einaudi, 1976.

ROMANELLI, S. (org.). Antologia Bilíngue. Clássicos da Língua Italiana. Tubarão: Copiart; 
Florianópolis: PGET/UFSC, 2012.

SOZZI, B. T. Polemiche cinquecentesche sulla lingua. In: MACHIAVELLI, N. Discorso o Dialogo Intorno alla Nostra Lingua. Torino: Einaudi, 1976, p.

SPERONI, S. Dialogo della Rettorica. A cura di Pozzi Mario. Roma: Vecchiarelli, 1978.

SPERONI, S. "Dialogo delle lingue". Tradução de Andreia Guerini, Anna Palma e Mauri Furlan. In: Mauri Furlan (Org.). Antologia Bilíngue. Clássicos da Teoria da Tradução. Vol. 4 Renascimento. Florianópolis: UFSC/NUPLITT, 2006, p. 220-261.

TESI, R. Storia dell'Italiano. La formazione della lingua comune dalle fasi iniziali al Rinascimento. Bologna: Zanichelli, 2007. 